\title{
Adaptation and Assessment of a High Resolution Semi-Discrete Numerical Scheme for Hyperbolic Systems with Source Terms and Stiffness
}

\author{
R. Naidoo ${ }^{1,2}$ and S. Baboolal ${ }^{2}$ \\ 1 Department of Mathematics and Physics, M.L. Sultan Technikon, P.O. Box 1334, \\ Durban 4000, South Africa \\ naidoor@yoda.cs.udw.ac.za \\ 2 Department of Computer Science, University of Durban-Westville, Private Bag \\ X54001, \\ Durban 4000, South Africa \\ sbab@pixie.udw.ac.za
}

\begin{abstract}
In this work we outline the details required in adapting the third-order semi-discrete numerical scheme of Kurganov and Levy [SIAM J. Sci. Comput. 22 (2000) 1461-1488.] to handle hyperbolic systems which include source terms. The performance of the scheme is then assessed against a fully discrete scheme, as well as reference solutions, on such problems as shock propagation in a Broadwell gas and shocks in gas dynamics with heat transfer.
\end{abstract}

\section{Introduction}

This paper is concerned with the numerical integration of

$$
\frac{\partial u(x, t)}{\partial t}+\frac{\partial f(u)}{\partial x}=\frac{1}{\varepsilon} g(u),
$$

a one-dimensional hyperbolic system of partial differential equations. Here $u(x, t)$ is the unknown m-dimensional vector function, $f(u)$ is the flux vector, $g(u)$ is a continuous source vector function on the right hand side (RHS), with $x$ the single spatial coordinate and $t$ the temporal coordinate and the parameter $\varepsilon>0$ distinguishes between stiff systems $(\varepsilon<<1)$ and standard, non-stiff ones $(\varepsilon=1)$.

Such equationss can be used to model many physical systems, including fluids and gases. In the last decade, particularly following the work of Nessyahu and Tadmor [1], a family of fully-discrete, high-resolution, Riemann-solver-free schemes have been produced in order to numerically solve hyperbolic systems such as the aforementioned. More recently, also based on the same Riemannsolver-free approach, second and third order semi-discrete schemes were devised by Kurganov and Tadmor [2] and Kurganov and Levy [3]. One advantage of the latter is that they can be applied on non-staggered grids and thus ease the implementation of boundary conditions. Here we are particularly interested in the details of adapting the latter so that it be can applied to systems with source 
terms including those that are stiff such as (1) above. In order to assess the performance of this scheme we examine its merits against an adaptation [4] following [5] for non-staggered grids, of the fully-discrete scheme of [1] for systems with source terms, as well as against exact or reference solutions for two prototype problems. One such is the problem of shock propagation in a Broadwell gas $[6,7]$ and the other is that of shocks in a model of gas dynamics with heat transfer.

\section{The modified numerical scheme}

\subsection{Derivation with source term}

Here the numerical integration of problem (1) is considered on some uniform spatial and temporal grids with the spacings, $\Delta x=x_{j+1}-x_{j} ; \Delta t=t^{n+1}-t^{n}$ (with $j$ and $n$ being suitable integer indices).

For the nonlinear homogeneous case of (1), Kurganov and Levy [3] obtain the third-order semi-discrete scheme

$$
\begin{aligned}
\frac{d \bar{u}_{j}}{d t} & =-\frac{1}{2 \Delta x}\left[f\left(u_{j+\frac{1}{2}}^{+}(t)\right)+f\left(u_{j+\frac{1}{2}}^{-}(t)\right)-f\left(u_{j-\frac{1}{2}}^{+}(t)\right)-f\left(u_{j-\frac{1}{2}}^{-}(t)\right)\right] \\
& -\frac{a_{j+\frac{1}{2}}(t)}{2 \Delta x}\left[u_{j+\frac{1}{2}}^{+}(t)-u_{j+\frac{1}{2}}^{-}(t)\right]-\frac{a_{j-\frac{1}{2}}(t)}{2 \Delta x}\left[u_{j-\frac{1}{2}}^{+}(t)-u_{j-\frac{1}{2}}^{-}(t)\right],
\end{aligned}
$$

where

$$
a_{j \pm \frac{1}{2}}^{n}=\max \left(\rho\left(\frac{\partial f}{\partial u}\left(u_{j \pm \frac{1}{2}}^{-}(t)\right)\right), \rho\left(\frac{\partial f}{\partial u}\left(u_{j \pm \frac{1}{2}}^{+}(t)\right)\right)\right),
$$

and

$$
u_{j \pm \frac{1}{2}}^{+}:=P_{j+1}\left(x_{j \pm \frac{1}{2}}, t^{n}\right) ; \quad u_{j \pm \frac{1}{2}}^{-}:=P_{j}\left(x_{ \pm \frac{1}{2}}, t^{n}\right) .
$$

In the above, the forms (4) are respectively the left and right intermediate values at $x_{j+\frac{1}{2}}$ of a piecewise polynomial interpolant $P_{j}\left(x, t^{n}\right)$ that fit an already computed or known cell average values $\left\{\bar{u}_{j}^{n}\right\}$ at time level $n$. Also $\rho($.$) denotes$ the spectral radius of the respective Jacobian, defining the maximum local propagation speeds $a_{j \pm \frac{1}{2}}^{n}$.

They also obtain an extension of the above when the RHS of (1) is of the form $\frac{\partial Q}{\partial x}$ where $Q\left(u(x, t), u_{x}(x, t)\right)$ is a dissipation flux satisfying a parabolicity condition [3].

However, to allow for an arbitrary source term, say, $g(u(x, t))$ in (1) (omitting for convenience the stiffness parameter) we must proceed as outlined in [3] and follow through the construction of the scheme with this added detail. Thus, employing the above mentioned uniform spatial and temporal grids and integrating (1) over the cell $I(x):=\left\{\xi|\xi-x| \leq \frac{\Delta x}{2}\right\}$ gives

$$
\bar{u}_{t}+\frac{1}{\Delta x}\left[f\left(u\left(x+\frac{\Delta x}{2}, t\right)\right)-f\left(u\left(x-\frac{\Delta x}{2}, t\right)\right)\right]=\bar{g}
$$


where

$$
\bar{u}(x, t):=\frac{1}{\Delta x} \int_{I(x)} u(\xi, t) d \xi
$$

and

$$
\bar{g}:=\frac{1}{\Delta x} \int_{I(x)} g(u(\xi, t)) d \xi
$$

Now assuming the $\left\{\bar{u}_{j}^{n}\right\}$ are already computed or known cell-averages of the approximate solution at time $t=t^{n}$ we integrate as in [3] over the control volumes $\left[x_{j-\frac{1}{2}, R}^{n}, x_{j-\frac{1}{2}, L}^{n}\right] \times\left[t^{n}, t^{n+1}\right],\left[x_{j-\frac{1}{2}, R}^{n}, x_{j+\frac{1}{2}, L}^{n}\right] \times\left[t^{n}, t^{n+1}\right]$ and $\left[x_{j+\frac{1}{2}, L}^{n}, x_{j+\frac{1}{2}, R}^{n}\right] \times$ $\left[t^{n}, t^{n+1}\right]$ where

$$
x_{j \pm \frac{1}{2}, L}^{n}:=x_{j \pm \frac{1}{2}}-a_{j \pm \frac{1}{2}}^{n} \Delta t ; \quad x_{j \pm \frac{1}{2}, R}^{n}:=x_{j \pm \frac{1}{2}}+a_{j \pm \frac{1}{2}}^{n} \Delta t
$$

with the piecewise polynomial form in the cell $I_{j}$ taken as

$$
P_{j}\left(x, t^{n}\right)=A_{j}+B_{j}\left(x-x_{j}\right)+\frac{1}{2} C_{j}\left(x-x_{j}\right)^{2} .
$$

where the constants $A_{j}, B_{j}, C_{j}$ are evaluated as in [3]. These then result respectively, in the weighted averages $\bar{w}_{j-\frac{1}{2}}^{n+1}, \bar{w}_{j}^{n+1}, \bar{w}_{j+\frac{1}{2}}^{n+1}$ which differ from those in [3] only in the respective additive source terms

$$
\begin{gathered}
\frac{1}{2 a_{j-\frac{1}{2}}^{n} \Delta t} \int_{x_{j-\frac{1}{2}}, L}^{x_{j-\frac{1}{2}}, R} \int_{t^{n}}^{t^{n+1}} g d x d t \\
\frac{1}{\Delta x-\Delta t\left(a_{j-\frac{1}{2}}^{n}+a_{j+\frac{1}{2}}^{n}\right)} \int_{x_{j-\frac{1}{2}}, R}^{x_{j+\frac{1}{2}}, L} \int_{t^{n}}^{t^{n+1}} g d x d t
\end{gathered}
$$

and

$$
\frac{1}{2 a_{j+\frac{1}{2}}^{n} \Delta t} \int_{x_{j+\frac{1}{2}}, L}^{x_{j+\frac{1}{2}}, R} \int_{t^{n}}^{t^{n+1}} g d x d t
$$

Then from the cell averages $\bar{w}_{j+\frac{1}{2}}^{n+1}$ and $\bar{w}_{j}^{n+1}$ are reconstructed third order piecewise polynomials [3] taken as

$$
\tilde{w}_{j \pm \frac{1}{2}}^{n+1}=\tilde{A}_{j \pm \frac{1}{2}}+\tilde{B}_{j \pm \frac{1}{2}}\left(x-x_{j \pm \frac{1}{2}}\right)+\frac{1}{2} \tilde{C}_{j \pm \frac{1}{2}}\left(x-x_{j \pm \frac{1}{2}}\right)^{2}, \quad \tilde{w}_{j}^{n+1}(x) \equiv \bar{w}_{j}^{n+1}
$$

where the constants $\tilde{A}_{j}, \tilde{B}_{j}$ and $\tilde{C}_{j}$ are evaluated as in [3]. The new cell averages on the unstaggered grids are obtained from these polynomials by [3]

$$
\bar{u}_{j}^{n+1}=\frac{1}{\Delta x}\left[\int_{x_{j-\frac{1}{2}}}^{x_{j-\frac{1}{2}, R}} \tilde{w}_{j-\frac{1}{2}}^{n+1} d x+\int_{x_{j-\frac{1}{2}, R}}^{x_{j+\frac{1}{2}, L}} \tilde{w}_{j}^{n+1} d x+\int_{x_{j+\frac{1}{2}, L}}^{x_{j+\frac{1}{2}}} \tilde{w}_{j+\frac{1}{2}}^{n+1} d x\right]
$$

The semi-discrete form is then defined by the limit 


$$
\frac{d \bar{u}_{j}(t)}{d t}=\lim _{\Delta t \rightarrow 0} \frac{\bar{u}_{j}^{n+1}-\bar{u}_{j}^{n}}{\Delta t} .
$$

Proceeding with (14) and (13) as in [3], the coefficients in the polynomial form simplify resulting in

$$
\begin{aligned}
& \frac{d \bar{u}_{j}}{d t}=-\frac{1}{2 \Delta x}\left[f\left(u_{j+\frac{1}{2}}^{+}(t)\right)+f\left(u_{j+\frac{1}{2}}^{-}(t)\right)-f\left(u_{j-\frac{1}{2}}^{+}(t)\right)-f\left(u_{j-\frac{1}{2}}^{-}(t)\right)\right] \\
&-\frac{a_{j+\frac{1}{2}}(t)}{2 \Delta x}\left[u_{j+\frac{1}{2}}^{+}(t)-u_{j+\frac{1}{2}}^{-}(t)\right]-\frac{a_{j-\frac{1}{2}}(t)}{2 \Delta x}\left[u_{j-\frac{1}{2}}^{+}(t)-u_{j-\frac{1}{2}}^{-}(t)\right] \\
&+\lim _{\Delta t \rightarrow 0} \frac{1}{2 \Delta x \Delta t} \int_{t^{n}}^{t^{n+1}} \int_{x_{j-\frac{1}{2}, L}^{j-\frac{1}{2}, R}}\left(t d x d t+\lim _{\Delta t \rightarrow 0} \frac{1}{2 \Delta x \Delta t} \int_{t^{n}}^{t^{n+1}} \int_{x_{j+\frac{1}{2}, L}}^{j+\frac{1}{2}, R} g d x d t\right. \\
&+\lim _{\Delta t \rightarrow 0} \frac{1}{\Delta t\left(\Delta x-\Delta t\left(a_{j+\frac{1}{2}}+a_{j-\frac{1}{2}}\right)\right)} \int_{t^{n}}^{t^{n+1}} \int_{x_{j-\frac{1}{2}, R}}^{j+\frac{1}{2}, L} g d x d t .
\end{aligned}
$$

We note that the non-smooth parts of the solution are contained over spatial widths of size $2 a_{j \pm \frac{1}{2}}^{n} \Delta t$. Full details with clear sketches are given in [3]. Now, when the limits are taken on the source integrals, the first two vanish as the Riemann fans shrink to zero. At the time, since $\bar{u}^{n}=\bar{u}(t)$ (and hence $g$ ) is a constant over this cell, it can be shown for the other that

$$
\lim _{\Delta t \rightarrow 0} \frac{1}{\Delta t\left(\Delta x-\Delta t\left(a_{j+\frac{1}{2}}+a_{j-\frac{1}{2}}\right)\right)} \int_{t^{n}}^{t^{n+1}} \int_{x_{j-\frac{1}{2}, R}}^{j+\frac{1}{2}, L} g d x d t=g\left(u_{j}^{n}\right) .
$$

Hence the modified semi-discrete scheme with source term $g(u(x, t))$ is

$$
\begin{array}{r}
\frac{d \bar{u}_{j}}{d t}=-\frac{1}{2 \Delta x}\left[f\left(u_{j+\frac{1}{2}}^{+}(t)\right)+f\left(u_{j+\frac{1}{2}}^{-}(t)\right)-f\left(u_{j-\frac{1}{2}}^{+}(t)\right)-f\left(u_{j-\frac{1}{2}}^{-}(t)\right)\right] \\
-\frac{a_{j+\frac{1}{2}}(t)}{2 \Delta x}\left[u_{j+\frac{1}{2}}^{+}(t)-u_{j+\frac{1}{2}}^{-}(t)\right]-\frac{a_{j-\frac{1}{2}}(t)}{2 \Delta x}\left[u_{j-\frac{1}{2}}^{+}(t)-u_{j-\frac{1}{2}}^{-}(t)\right]+g\left(u_{j}(t)\right) .
\end{array}
$$

where the rest of the terms are as in (2)-(3).

To compute with (17), it is convenient to use ODE system solvers, such as Runge-Kutta formulae. For instance, writing (17) in the form,

$$
\frac{d u_{j}}{d t}=F_{j}
$$

where $F_{j}$ is the vector of the RHS, we can employ the second-order (in time) Runge-Kutta (RK2) scheme [2] for it as:

$$
R K 2:\left\{\begin{array}{l}
U^{(1)}=U^{n}+\Delta t F\left(U^{n}\right) \\
U^{(2)}=\frac{1}{2} U^{n}+\frac{1}{2}\left[U^{(1)}+\Delta t F\left(U^{(1)}\right)\right] \\
U^{n+1}=U^{(2)}
\end{array}\right.
$$


where the $U$ denotes the vector of components $u_{j}$, the superscript $n$ and $n+1$ denote successive time levels, whilst the other $(1,2)$ denote intermediate values.

We shall refer to the scheme (17) with RK2 (19) as the SD3 scheme. We note that such a scheme is generally third order except in regions of steep gradients when it degrades to order two [3]. Since also, RK2 is second order in time, it will make sense when we compare its performance to that of the fully discrete scheme (NNT) for systems with source terms for integration on unstaggered grids [4]:

$$
\begin{aligned}
\bar{u}_{j}^{n+1}= & \frac{1}{4}\left(\bar{u}_{j+1}^{n}+2 \bar{u}_{j}^{n}+\bar{u}_{j-1}^{n}\right)-\frac{1}{16}\left(u_{x j+1}^{n}-u_{x j-1}^{n}\right)-\frac{1}{8}\left[u_{x j+\frac{1}{2}}^{n+1}-u_{x j-\frac{1}{2}}^{n+1}\right] \\
& +\frac{\Delta t}{8}\left[g\left(u_{j+1}^{n}\right)+2 g\left(u_{j}^{n}\right)+g\left(u_{j-1}^{n}\right)+g\left(u_{j+1}^{n+1}\right)+2 g\left(u_{j}^{n+1}\right)+g\left(u_{j-1}^{n+1}\right)\right] \\
& -\frac{\lambda}{4}\left[\left(f_{j+1}^{n}-f_{j-1}^{n}\right)+\left(f_{j+1}^{n+1}-f_{j-1}^{n+1}\right)\right]
\end{aligned}
$$

where $\lambda=\Delta t / \Delta x$ and which will be used used with an UNO derivative approximation $[1,4]$.

\subsection{Implementation details}

The implementation of the NNT scheme (20) above follows previous reports [1, 4], where in particular we mention that the source term can make the scheme implicit. The latter then requires an iteration at each grid point at every time level. The implementation of the modified semi-discrete scheme SD3 (17) follows closely the prescription given in [3] where in particular we employ the constants given in their equation (2.9) for the non-oscillatory piece-wise polynomial (9). In addition, it is required to compute at every time step the spectral radii (3) of the Jacobians of the flux terms, which we obtained exactly for the small test systems to follow. Finally, with chosen initial and boundary conditions, the solution is advanced with the explicit Runge-Kutta scheme (18)-(19). The codes were written in 64-bit real precision Fortran 77, employing real-time graphics to depict evolving profiles. They were compiled with the Salford FTN95/win32 version 1.28 compiler and run on a PC under MS Windows 2000 and NT.

\section{Applications and tests}

\subsection{Shocks in a Broadwell gas}

Here we solve the governing equations for a Broadwell gas $[6,7]$,

$$
\begin{gathered}
\frac{\partial \rho}{\partial t}+\frac{\partial m}{\partial x}=0 \\
\frac{\partial m}{\partial t}+\frac{\partial z}{\partial x}=0 \\
\frac{\partial z}{\partial t}+\frac{\partial m}{\partial x}=\frac{1}{\varepsilon}\left(\rho^{2}+m^{2}-2 \rho z\right),
\end{gathered}
$$


where $\varepsilon$ is the mean free path and $\rho(x, t), m(x, t), z(x, t)$ are the density, momentum and flux respectively. The range $\varepsilon=1 . .10^{-8}$ cover the regime from the non-stiff to the highly stiff. In particular, the limit $\varepsilon=10^{-8}$ requires a renormalization of the variables such as in the form

$$
\bar{x}=\frac{1}{\varepsilon} x, \quad \bar{t}=\frac{1}{\varepsilon} t
$$

followed by computations on an equivalent finer grid (see for example [8]).

We observe that in the limit $\varepsilon \rightarrow 0$ we arrive at

$$
z=z_{E}(\rho, m)=\frac{1}{2 \rho}\left(\rho^{2}+m^{2}\right)
$$

which leads to the equilibrium solution of the governing equations above which then reduce to the Euler equations.

The SD3 (17) and NNT scheme (20) were applied to the above with the two sets (Rim1 and Rim2) of initial conditions corresponding to several Riemann problems, each distinguished by a specific $\varepsilon$-value:

$$
\begin{gathered}
\operatorname{Rim} 1:\left\{\begin{array}{l}
\rho=2, m=1, z=1 ; \quad x<x_{J} . \\
\rho=1, m=0.13962, z=1 ; \quad x>x_{J} .
\end{array}\right. \\
\operatorname{Rim} 2:\left\{\begin{array}{l}
\rho=1, m=0, z=1 ; \quad x<x_{J} . \\
\rho=0.2, m=0, z=1 ; \quad x>x_{J} .
\end{array}\right.
\end{gathered}
$$

In all calculations absorbing boundary conditions were employed, where in particular, the boundary values were obtained by quadratic extensions of internal point values on a fixed spatial grid, over an integration domain on the $X$-axis. Results obtained are depicted in Figure 1.

Other parameters used here were $\Delta x=0.01, \Delta t=0.005, x_{J}=5$ in (a) and (b) and $\Delta x=0.02, \Delta t=0.001, x_{J}=10$ in (c) and (d) for both methods. We observe that in virtually all cases, the semi-discrete scheme give better results than the modified NNT scheme.

\subsection{Shocks in an Eulerian gas with heat transfer}

Here we solve the Euler equations for the one-dimensional flow of a gas in contact with a constant temperature bath [9]:

$$
\begin{gathered}
\frac{\partial \rho}{\partial t}+\frac{\partial(\rho u)}{\partial x}=0 \\
\frac{\partial(\rho u)}{\partial t}+\frac{\partial\left(\rho u^{2}+p\right)}{\partial x}=0 \\
\frac{\partial(\rho E)}{\partial t}+\frac{\partial(\rho u E+u p)}{\partial x}=-K \rho\left(T-T_{0}\right) .
\end{gathered}
$$



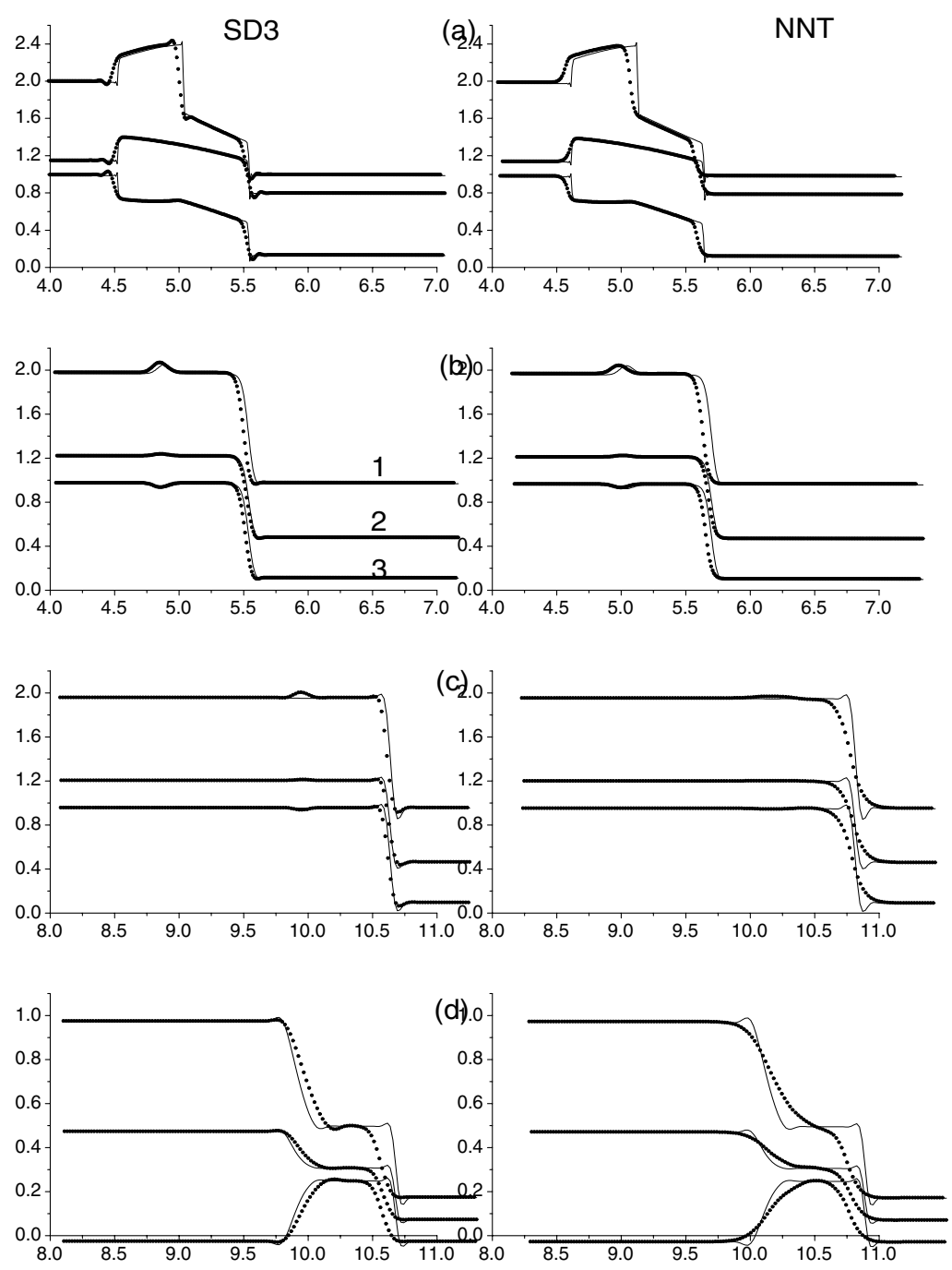

Fig. 1. Broadwell gas shock solutions with (a) $\varepsilon=1$ (Rim1), (b) $\varepsilon=0.02$ (Rim1), (c) $\varepsilon=10^{-8}$ (Rim 1$)$ and (d) $\varepsilon=10^{-8}$ (Rim2). Here the curve labelled $1 \sim \rho, 2 \sim z$, $3 \sim m$. The snap-shot time is $t=0.5$ in all cases. The dotted lines are computed solutions and the solid ones are 'exact' (refined grid) solutions. 
The $\rho, u, T, e, E=e+\frac{1}{2} u^{2}, p=(\gamma-1) \rho e$ are the density, flow velocity, temperature in units of $e$, internal energy, total energy and pressure respectively with $K>0$ the heat transfer coefficient and $T_{0}$ the constant bath temperature.

The initial conditions used were:

$$
\operatorname{Rim} 3: \begin{cases}\rho=2.5, u=1.0, p=1.0 ; & x<50 . \\ \rho=1.0, u=0.4, p=0.4 ; & x>50 .\end{cases}
$$

where different $K=1,50,400,1000$ are employed. Computed results with SD3 and NNT are shown in Figure 2.
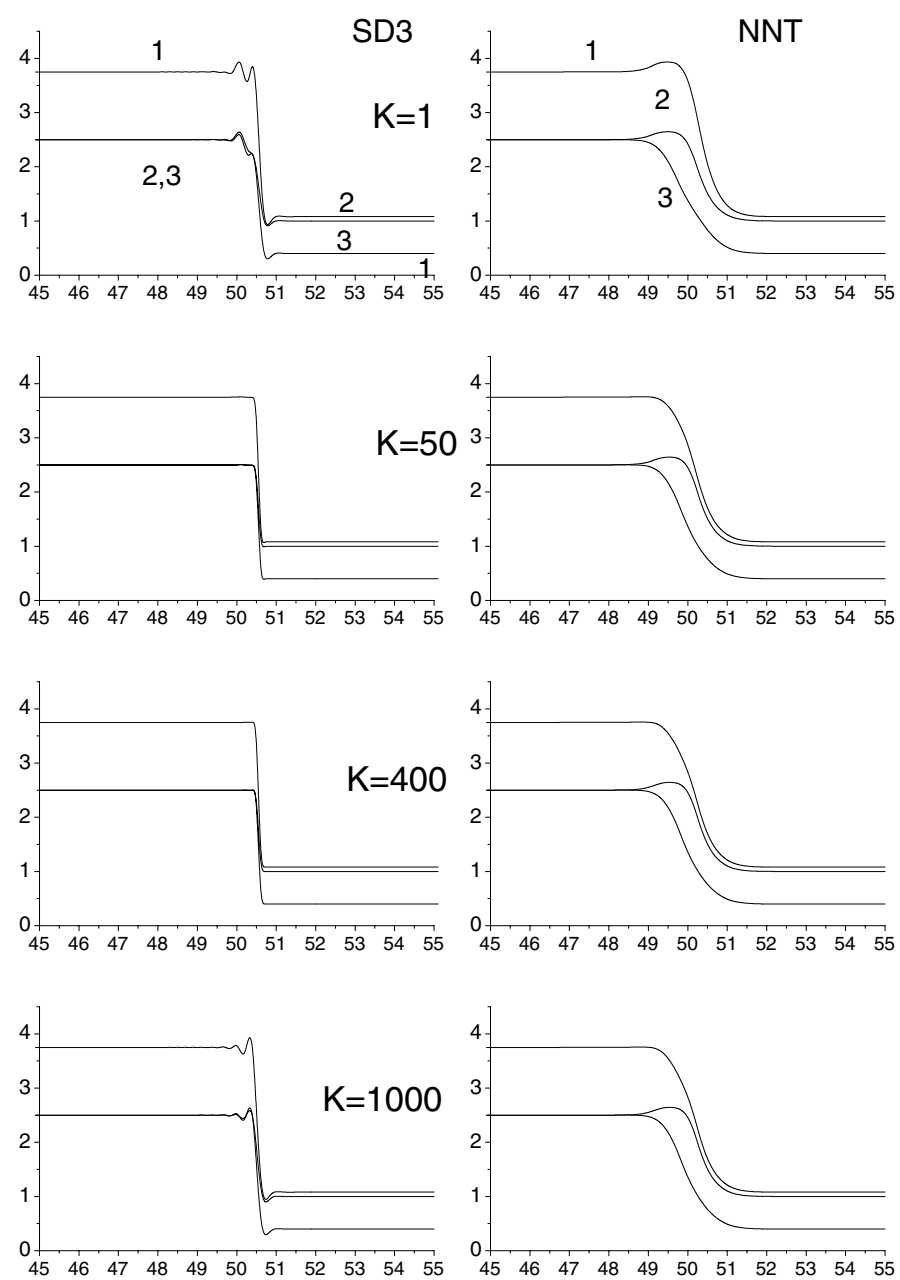

Fig. 2. Shocks in a gas with heat transfer. The curve labelled $1 \sim \rho E, 2 \sim \rho, 3 \sim \rho u$. The grid lengths of $\Delta x=0.1$ and $\Delta t=0.001$ were used for both methods. The output time is $t=0.4$ in all cases. 
In these we observe SD3 captures the shocks significantly better than does NNT. Although the 'exact' (grid refined ones) are not shown here we mention that they are close to the SD3 ones, except for the oscillations seen in them. We believe the oscillations are due to the derivative calculations from the fitted piecewise polynomials, and can be improved by taking different smoothing coefficients, as suggested in [3]. Further, the NNT curves show poor resolution of the shocks in comparison, and also show far more dissipation, as expected [3].

\section{Conclusion}

We have indicated in this work, how the third-order semi-discrete numerical scheme of Kurganov and Levy [3] can be suitably modified to include source terms in one-dimensional hyperbolic systems. Results obtained with it on shock propagation in a Broadwell gas and in a gas dynamics model with heat transfer show its clear superiority over a fully discrete modification for systems with source terms [4].

\section{Acknowledgements}

The authors wish to thank Professors D. Levy, G. Russo and Shi Jin for their helpful correspondences.

\section{References}

1. Nessyahu, H., Tadmor, E.: Non-oscillatory central differencing for hyperbolic conservation laws. J. Comput.Phys. 87 (1990) 408-463.

2. Kurganov, A., Tadmor, E.: New high-resolution central schemes for nonlinear conservation laws and convection-diffusion equations. J. Comput. Phys. 160 (2000) 241-282.

3. Kurganov, A., Levy, D.: A third-order semi-discrete central scheme for conservation laws and convection-diffusion equations. SIAM J. Sci. Comput. 22 (2000) 1461-1488.

4. Naidoo, R., Baboolal, S.: Modification of the second-order Nessyahu-Tadmor central scheme to a non-staggered scheme for hyperbolic systems with source terms and its assessment. Submitted to SIAM J. Sci. Comput.

5. Jiang, G.-S., Levy, D., Lin, C.-T., Osher, S., Tadmor, E.: High-resolution nonoscillatory central schemes with non-staggered grids for hyperbolic conservation laws. SIAM J. Numer. Anal. 35 (1998) 2147-2168.

6. Caflisch, R.E., Jin, S., Russo, G.: Uniformly accurate schemes for hyperbolic systems with relaxation. SIAM J. Numer. Anal. 34 (1997) 246-281.

7. Jin, S.: Runge-Kutta methods for hyperbolic conservation laws with stiff relaxation terms. J. Comput. Phys. 122 (1995) 51-67.

8. Naldi, G., Pareschi, L.: Numerical schemes for hyperbolic systems of conservation laws with stiff diffusive relaxation. SIAM J. Numer. Anal. 37 (2000) 1246-1270.

9. Pember, R. B.: Numerical methods for hyperbolic conservation laws with stiff relaxation II. Higher-order Godunov methods. SIAM J. Sci. Comput. 14 (1993) 824-829. 